\title{
Stop Climate Change
}

\section{Khalidullin 0*}

Kazakh National University, Russia

*Corresponding author: Oleg Halidullin, Ecology Professor, Kazakh National University, Russia, Tel: 87770550099; Email: 7115215@mail.ru

\section{Editorial}

Volume 4 Issue 3

Received Date: June 11, 2020

Published Date: July 02, 2020

DOI: $10.23880 /$ ijoac- 16000191

\section{Editorial}

Your idea, of course, is crazy. The whole question is whether she is crazy enough to be true.-

Niels Bohr

In the article "Who is behind Greta Tunberg," Victor Savin argues that $70-80 \%$ of the contribution to the greenhouse effect comes from ordinary water vapor. The achievements of scientists on global warming are not in doubt, however, is $\mathrm{CO}_{2}$ the main insulation? By its quantitative composition in the atmosphere it is 26 times less than water vapor. This is according to classic data. Let's look at the evaporation of water. Water, in its basic functions, supplies plants and animals with trace elements dissolved underground and along river beds. Entering the bodies of organisms and plant roots in the form of a solution, water is repeatedly converted into blood, lymph, sweat, urine, many other organs of the body, plant juices, and enters the atmosphere in the form of exhalation, secretions, transpiration - in a completely different quality. The sum of all these purely individual vapors forms a substance in the atmosphere in the form of clouds, with certain properties polished over millions of years, which precipitate in beaten paths at predetermined intervals in specified places on the planet. It is these properties that have created various habitats from deserts to the tropics. A variety of secretions can easily be imagined if we recall that the smell is formed by the molecules inherent in each species of animals and plants and within each individual.

The properties of clouds are their saturation, physical and chemical composition, electric charges, specific gravity, height above the planet's surface, volume, time spent in the atmosphere. Temperature, pressure in the atmosphere and in the surface layer depend on this, and a precipitation mechanism is created that strictly determines where, how much and when to give moisture to every place on the planet.

We took almost all of the land from nature by cutting down forests, plowing the soil, flooding it with water in front of hydroelectric power stations, using water unnaturally we turned it into a working reagent. We move it with pumps, boil, heat, compress, cool, spray, fill the fields, destroy its molecules in the air with engines, furnaces, compressors, everything that surrounds us and ourselves.

Vapors from all these processes do not have the properties inherent in vapors from living organisms and plants - these are artificial vapors. The physical, chemical and all other parameters of the molecules have changed. A new substance in the clouds produces, concentrates vapors in a new way. A new mechanism of atmospheric phenomena is created with a change in pressure, temperature, movement of air masses, all other driving forces. The distribution, volumes and time parameters of precipitation depend on this, and the places of their precipitation change. Whether $\mathrm{CO} 2$ is involved in this mechanism and what its role is here is a question. But each of us sees and feels the impact of heavy clouds, which increasingly block the sun from us and masses of water pour out from these clouds, which flood us with floods in some places and, on the contrary, do not give water in others, leading to dry areas and fires.

The volumes of artificial fumes with the development of industry and human comfort have become comparable with the volumes of organic fumes. Humanity has destroyed the effects of organic fumes on 70 percent of the land. From these places, water began to evaporate, which did not pass the path indicated by nature - to deliver nutrients to living organisms and plants. In total, we take 22 cubic kilometers of water from open and underground sources. These waters lose their natural functions and evaporate without changing the structure [1]. Water completes its idle cycle.

All the parameters of gaseous water and its drops have changed, which have led to a change in the quality of the clouds themselves, including the location in height in the atmosphere. Weighted fumes cannot rise to a predetermined 
height. Therefore, the clouds became more massive, slower in speed of movement. Perhaps the vapor in the molecular state rose higher and moved further along other routes. The time spent by the water in the clouds has changed. All this in the complex has changed the place of precipitation, their volume and schedule. From here, we observe floods in some areas, droughts in others, and the extinction of species of insects and animals. The decrease in transit in the range and height of the movement of clouds led to a decrease in precipitation on the glaciers of the polar poles and mountain peaks. Precipitation on the way to these places does not accumulate in glaciers, but replenish the oceans with additional volumes. Therefore, the level of the oceans rises and the ice layer of the glaciers decreases.

There is numerical evidence that carbon dioxide raises into the atmosphere 108 times less than artificial fumes from technological processes alone - less than $1 \%$. This hypothesis requires scientific evidence, which should lead to a new strategy. It should be based on the all-round return to nature of ruined territories - arable land and irrigated fields, landfills and ore landfills, areas of man-made reservoirs, asphalt and concrete pavements. To this trace there is no need to add total water savings at all enterprises of agricultural, municipal, industrial and all other types of human activity. Nature will return its natural process of the water cycle. There are such opportunities if we use the successes of developed countries in environmental protection. It is known that in the Scandinavian countries there are no more landfills; in Israel, plant products are grown with minimum water consumption. Famous houses with vertical and roof landscaping. Known inventions that allow you to generate electricity without reservoirs. There are ways to economically consume water on airplanes and trains. Such and other measures of total water saving should be applied in all cities and countries by the entire population of the planet.

Only returning it to nature, millions of years of proven technology of the water cycle, can reduce natural disasters and stop climate change. To prove the provisions of the Hypothesis, studies of its basic elements are necessary. To do this, a new team of new specialists is needed, which will be able to bring the scientific base and turn the consciousness of the population turned to carbon dioxide propaganda.

\section{Reference}

1. Khalidullin $O$ (2019) Slavery of Water and Climate. Acta Scientific Microbiology (ASMI) S1: 31-35.

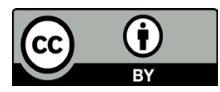

